\title{
Dihydromyricetin Alleviates High Glucose-Induced Oxidative Stress and Apoptosis in Human Retinal Pigment Epithelial Cells by Downregulating miR-34a Expression
}

This article was published in the following Dove Press journal:

Diabetes, Metabolic Syndrome and Obesity: Targets and Therapy

\begin{abstract}
Wenjun $\mathrm{Li}^{\prime}$
Hongxia Xiao ${ }^{2}$

'Department of Ophthalmology, NHC Key Laboratory of Hormones and Development, Tianjin Key Laboratory of Metabolic Diseases, Chu Hsien-I Memorial Hospital \& Tianjin Institute of Endocrinology, Tianjin Medical University, Tianjin 300134, People's Republic of China; ${ }^{2}$ Department of Ophthalmology, Jingmen No. 2 People's Hospital, Jingmen 448000, People's Republic of China
\end{abstract}

Background: Diabetic retinopathy (DR) is one of the most common microvascular complications of diabetes mellitus, which leads to neuronal and vascular dysfunction in the retina with a final outcome of complete loss of vision. The aim of the present study was to investigate the effects of dihydromyricetin (DHM), a natural flavanol compound, on diabetic retinopathy (DR) and identify its potential mechanisms.

Methods: Retinal pigment epithelial cell line (ARPE-19) treated with high glucose (HG) was used to simulate the DR model in vitro. After treatment with different concentrations of DHM, the cell viability, production of reactive oxygen species (ROS) and the levels of oxidative stress-related markers in the in vitro model were detected using corresponding kits. Cell apoptosis was determined using terminal-deoxynucleotidyl transferase mediated nick end labeling (TUNEL) staining, and the expression of apoptotic proteins was examined using Western blot analysis. Subsequently, microRNA (miR)-34a expression was measured by reverse transcription-quantitative PCR (RT-qPCR). The levels of oxidative stress and apoptosis were evaluated after miR-34a overexpression.

Results: Results indicated that DHM dose-dependently elevated the decreased cell viability induced by HG. Moreover, the content of ROS was significantly reduced in HG-stimulated ARPE-19 cells, accompanied by enhanced activities of superoxide dismutase (SOD) and catalase (CAT) antioxidases, as well as concentration of glutathione (GSH). Furthermore, remarkably decreased apoptosis of ARPE-19 cells induced by HG was observed following DHM intervention. Importantly, HG stimulation notably upregulated miR-34a expression, which was reversed by DHM treatment. Importantly, the inhibitory effects of DHM on HGinduced oxidative stress and apoptosis of ARPE-19 cells were restored following miR-34a overexpression.

Conclusion: Taken together, this work demonstrated that DHM exerts protective effects on HG-induced oxidative stress and apoptotic damage in ARPE-19 cells via inhibition of miR34a expression, providing a promising therapeutic agent for the treatment of DR.

Keywords: diabetic retinopathy, high glucose, oxidative stress, dihydromyricetin, apoptosis

\section{Introduction}

Diabetic retinopathy (DR), a chronic and progressive microvascular complication of diabetes mellitus, is a leading cause of preventable blindness worldwide that seriously degrades the life quality of human beings. ${ }^{1}$ At present, the prevalence of diabetes in China is approximately $5.6 \% .^{2}$ The morbidity of DR increases during
Correspondence: Hongxia Xiao Jingmen No. 2 People's Hospital, 39 Xiangshan Avenue, Jingmen, Hubei 448000, People's Republic of China Email drhongxia@I63.com
Diabetes, Metabolic Syndrome and Obesity: Targets and Therapy 2021:14 387-397 
the progression of diabetes, and it has been reported that half of the diabetic patients with a course of 10-20 years will develop DR complications, and that DR can definitely complicate the symptoms of diabetic patients with a course of more than 20 years. $^{3}$ With the in-depth study and clinical applications of medicine, treatment methods of DR have constantly improved. So far, however, there has been no effective treatment for this disease yet. ${ }^{4}$ Therefore, it is of utmost importance for us to understand the underlying mechanisms and identify therapeutic agents associated with DR.

In the past decades, research on the pathophysiology and management of DR have revolutionized the understanding of this disease. The retinal pigment epithelium (RPE), a highly specialized pigmented layer, is a gatekeeper of the outer retina, which not only provides mechanical support, but also regulates the metabolism of the photoreceptors through controlling the selective molecular transport between outer retina and the choroidal blood. ${ }^{5,6}$ In addition to imparting a physical barrier, RPE is also essential for the development and maintenance of adjacent photoreceptors in the retina. ${ }^{7}$ RPE cells are one of the most vulnerable cellular populations of blood-retinal barrier. A growing body of literature has shown that the glucose-stimulated RPE cells have been widely used as in vitro models for DR research. ${ }^{8,9}$ Increasing evidence shows that prolonged elevation of glucose added in RPE cells can trigger oxidative stress by inducing the production of intracellular reactive oxygen species (ROS). ${ }^{10,11}$ The accumulation of intracellular ROS activates a cascade of biochemical and molecular events and results in the apoptosis of RPE cells, further leading to the destruction of the retinal environment and the occurrence of retinopathy damage. ${ }^{12,13}$ Thus, the suppression of oxidative stress and apoptosis has been suggested to be a potentially promising strategy for the treatment of DR.

Dihydromyricetin (DHM) is a natural flavanol compound isolated from Ampelopsis grossedentata, which has been shown to possess a variety of pharmacological properties such as anti-oxidative, anti-inflammatory and anti-tumor effects. ${ }^{14-17}$ A report demonstrated previously that DHM ameliorated oxidative stress and apoptotic damage of human umbilical vein endothelial cells induced by sodium nitroprusside. ${ }^{18}$ Jiang et al found that DHM protected PC12 cells against oxidative stress response induced by methylglyoxal through regulating the AMPK/ GLUT4 pathway. ${ }^{19}$ Importantly, DHM enhanced glucose uptake in 3T3-L1 cells, and prevented the development of diabetic cardiomyopathy and diabetic nephropathy. ${ }^{20-22}$ Therefore, we speculated that DHM might exert protective effects in DR by a certain mechanism.

MicroRNAs (MiRNAs), a class of small non-coding RNAs with 19-24 nucleotides in length, exert crucial regulatory effects in the progression of DR. ${ }^{23,24}$ Existing study has shown that microRNA (miR)-34a expression was markedly upregulated in retinal endothelial cells cultured with high glucose (HG), and P53/miR-34a inhibition improved diabetic endothelial dysfunction by activating SIRT1. ${ }^{25,26}$ Moreover, DHM was reported to suppress diabetic cardiomyopathy via miR-34a inhibition through the autophagy activation. ${ }^{21}$ Therefore, whether DHM could alleviate HG-induced oxidative stress and apoptosis of RPE cells by regulation miR-34aexpression aroused our research interest.

Based on the above literature, this study aimed to investigate the effects and potential mechanism of DHM on HG-induced oxidative stress and apoptosis in human retinal epithelial cell line ARPE-19 cells. Our findings may identify a novel pharmaceutical approach for the treatment of DR.

\section{Materials and Methods Cell Culture and Treatment}

ARPE-19 cells were obtained from American Type Culture Collection (ATCC, Manassas, VA). The cells were maintained in Dulbecco's Modified Eagle Medium (DMEM; Gibco; Thermo Fisher Scientific, Inc.) containing 10\% fetal bovine serum (FBS; Gibco; Thermo Fisher Scientific, Inc.) in a humidified incubator at $37^{\circ} \mathrm{C}$ with $5 \%$ $\mathrm{CO}_{2}$. ARPE-19 cells were exposed to medium supplemented with normal glucose ( $5.5 \mathrm{mmol} / \mathrm{L}$ glucose, NG group), $5.5 \mathrm{mmol} / \mathrm{L}$ glucose $+22.5 \mathrm{mmol} / \mathrm{L}$ mannitol (MA group) or high glucose $\left(25 \mathrm{mmol} / \mathrm{L}\right.$ glucose, $\mathrm{HG}$ group) at $37^{\circ} \mathrm{C}$ for $48 \mathrm{~h}$. In the DHM-treated group, ARPE-19 cells were pretreated with 30, 100 and $300 \mu \mathrm{mol} / \mathrm{L}$ of DHM (Lyphar Biotech Co., Ltd, Xi'an, China) for $3 \mathrm{~h}$ prior to highglucose treatment and DHM was washed out after pretreatment.

\section{Cell Transfection}

For cell transfection, ARPE-19 cells $\left(1 \times 10^{6}\right.$ cells/well $)$ were seeded into a 6-well plate, and transfection was conducted when cells in the logarithmic growth phase reached $80 \%$ confluence. MiR-34a mimic and its negative control (mimic-NC) were designed and synthesized 
by GenePharma (Shanghai, China). Transfection experiments were performed using Lipofectamine ${ }^{\circledR} 2000$ (Invitrogen; Thermo Fisher Scientific, Inc.) according to the standard protocol. Cells were harvested 48 $\mathrm{h}$ following transfection and the transfection efficiency was determined using reverse transcription-quantitative PCR (RT-qPCR).

\section{Cell Viability Assay}

ARPE-19 cells (5000 cells/well) in the logarithmic growth phase were seeded into a 96-well microplate and cultured overnight to allow cells to attach before further treatment. Cell viability was evaluated using a Cell Counting Kit-8 (CCK-8) kit obtained from Shanghai Yi Sheng Biotechnology Co., Ltd (Shanghai, China). Briefly, after treatment, $10 \mu \mathrm{L}$ of CCK-8 was added to each well and incubated at $37^{\circ} \mathrm{C}$ for $2 \mathrm{~h}$. The optical density was determined at $450 \mathrm{~nm}$ using a microplate reader (Bio-Rad Laboratories, Inc.).

\section{Detection of Intracellular ROS}

An ROS assay kit (Beyotime Biotechnology, Shanghai, China) with cell-permeable 2', 7'-dichlorodihydrofluorescein diacetate (DCFH-DA) was used to detect the production of intracellular ROS in ARPE-19 cells. In brief, cells were plated in a 6-well plate. DCFH-DA $(10 \mu \mathrm{mol} / \mathrm{L})$ and e 4 '6-diamidino-2-phenylindole (DAPI; $1 \mu \mathrm{g} / \mathrm{L}$ ) were added to the well and incubated for $15 \mathrm{~min}$ at $37^{\circ} \mathrm{C}$. The images were captured using a confocal microscope (Olympus Corporation, Tokyo, Japan).

\section{Test for the Oxidative Stress-Related Factors}

To evaluate the levels of oxidative stress, oxidative stress biomarkers including glutathione (GSH), superoxide dismutase (SOD) and catalase (CAT) were evaluated using commercial kits in accordance with the specification provided by the supplier. Above kits were obtained from Nanjing Jiancheng Bioengineering Institute (Nanjing, China).

\section{Terminal-Deoxynucleoitidyl Transferase Mediated Nick End Labeling (TUNEL) Staining}

Apoptosis of ARPE-19 cells was determined using the TUNEL assay kit purchased from Beyotime Biotechnology (Shanghai, China) according to the standard protocol. In brief, cells were collected and fixed with $4 \%$ paraformaldehyde at room temperature for $25 \mathrm{~min}$. Subsequently, cell membranes were permeabilized with the phosphate buffer solution (PBS) containing $0.3 \%$ Triton $\mathrm{X}-100$ for $5 \mathrm{~min}$ at room temperature. The fluorescein-dUTP was then added to cells to stain the nuclei in the apoptotic cells, and DAPI (5 $\mathrm{mg} / \mathrm{mL}$ ) was utilized for staining the cell nuclei. The TUNEL-positive cells were visualized using a fluorescence microscope (Olympus Corporation, Tokyo, Japan). Five random separate fields were employed to calculate the number of TUNEL-positive cells.

\section{RT-qPCR Analysis}

Total RNA was extracted from cells using TRIzol ${ }^{\circledR}$ (Thermo Fisher Scientific, Inc) and processed as recommended by the manufacturer's specifications. Subsequently, complementary DNA (cDNA) was synthesized using the obtained total RNA with a reverse transcription kit (Beijing Transgen Biotech Co., Ltd., China). qPCR was performed using SYBR Green Supermix (BioRad Laboratories, Inc.) on the ABI 7500 system (Applied Biosystems; Thermo Fisher Scientific, Inc.). Comparative mRNA levels of target genes were calculated by the $2^{-\Delta \Delta \mathrm{Cq}}$ method. U6 and GADPH were used as internal standards.

\section{Western Blot Analysis}

For the isolation of proteins, cells were lysed in ice-cold cell extraction buffer (Beyotime Institute of Biotechnology). Then, the concentration of protein was quantified by the bicinchoninic acid (BCA) protein assay (Beyotime Institute of Biotechnology). A total of $40 \mu \mathrm{g}$ protein was subjected to $10 \%$ sodium dodecyl sulfate polyacrylamide gel electrophoresis (SDS-PAGE) and transferred to a nitrocellulose blotting membrane (GE Healthcare Life Science, Germany). Then, the membrane was blocked with $5 \%$ skim milk at room temperature for $1.5 \mathrm{~h}$. Subsequently, the transferred membranes were incubated with the primary antibodies at $4^{\circ} \mathrm{C}$ overnight. After being washed three times with TBST, these blots were incubated with HRP-conjugated secondary antibody at room temperature for $1 \mathrm{~h}$. The immunoreactive protein bands on the membranes were visualized using the Odyssey Infrared Imaging System (LI-COR Biosciences). GAPDH was used for normalization. Anti-Bcl-2 (cat. no 4223T), anti-Bax (cat. no 
5023T), anti-cleaved caspase-3 (cat. no 9664S), anticleaved caspase-9 (cat. no 20750S) and antiglyceraldehyde 3-phosphate dehydrogenase (GAPDH; cat. no 5174S) antibodies were the products of Cell Signaling Technology, Inc. (Boston, MA, USA).

\section{Statistical Analysis}

Three biological replicates were included in each experiment. Data were presented as the mean \pm standard deviation. One-way analysis of variance was used to assess multiple differences, followed by a Tukey's post hoc test with SPSS 19.0 software (IBM Corp., Armonk, NY, USA).
$\mathrm{P}<0.05$ was considered to indicate a statistically significant difference.

\section{Results}

\section{DHM Alleviates Oxidative Stress in HG-Induced ARPE 19 Cells}

To study the effects of DHM on DR in vitro, cell viability was firstly determined using a CCK-8 kit to evaluate the cytotoxicity of DHM on ARPE 19 cells. Results displayed in Figure $1 \mathrm{~A}$ indicated that there was no significant difference in DHM-treated ARPE 19 cells compared with the untreated group. Then, HG
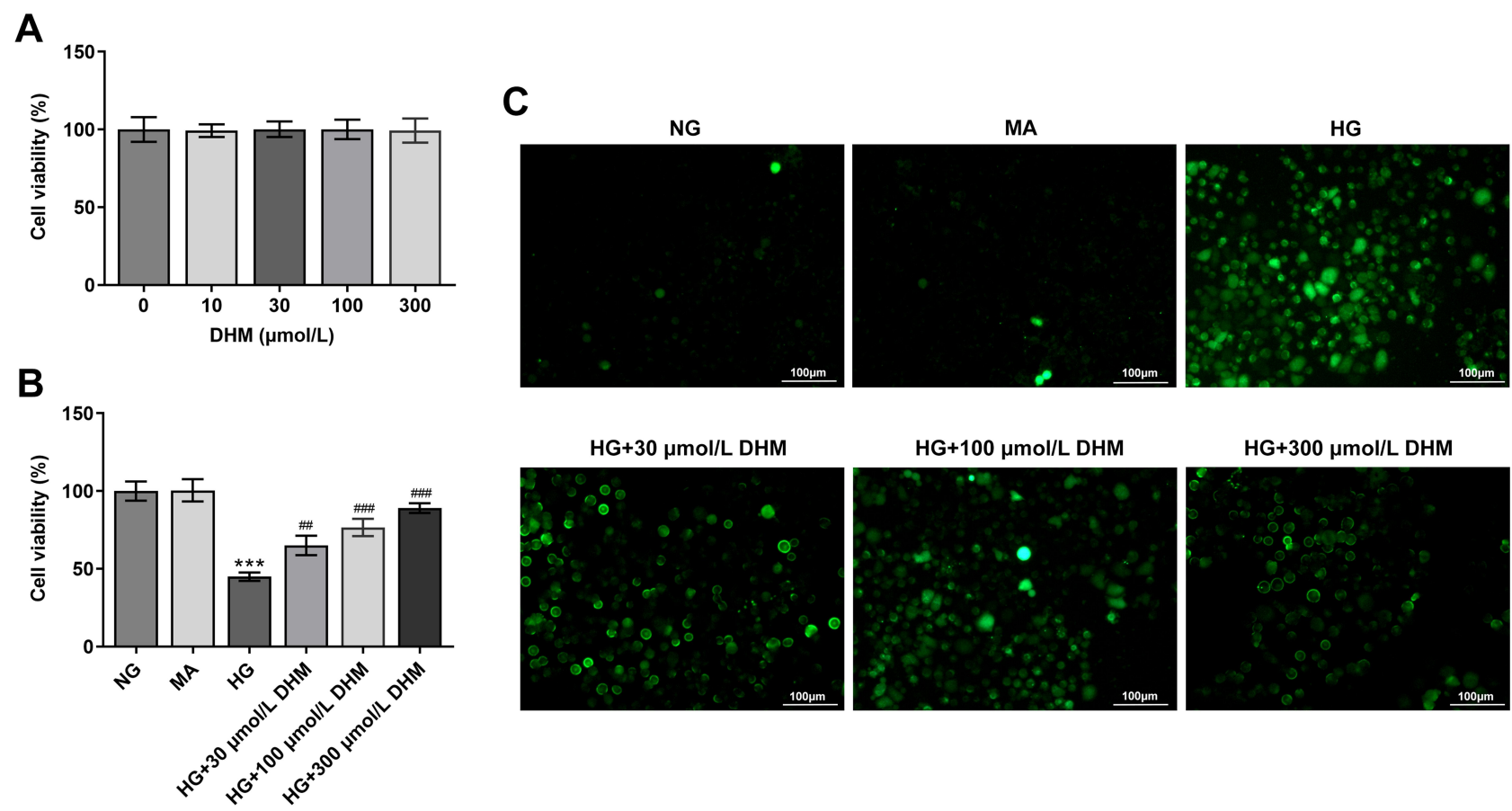

D
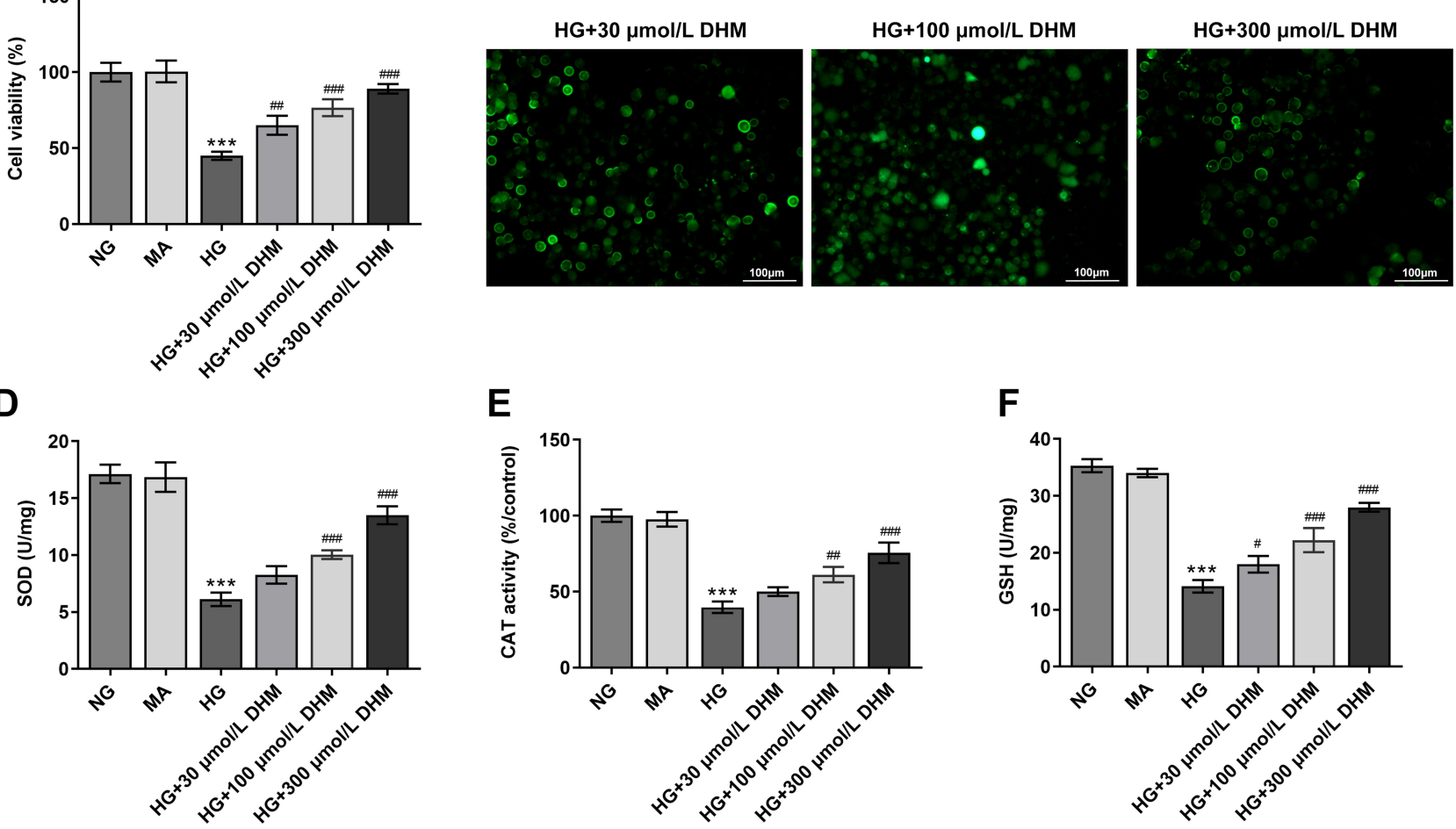

E

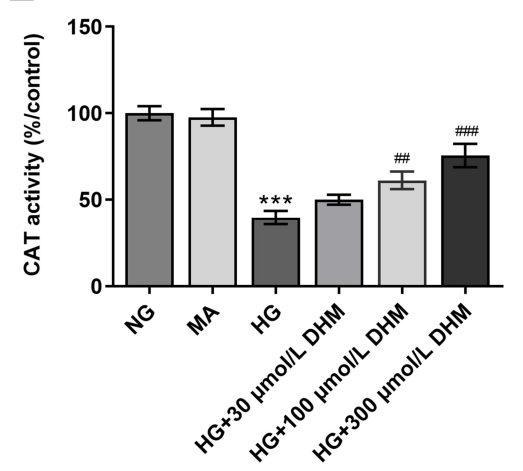

$\mathbf{F}$

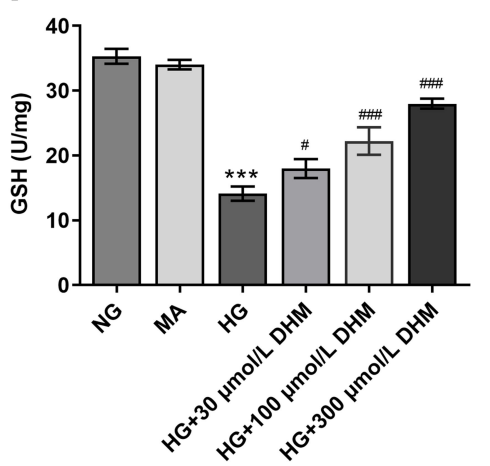

Figure I DHM attenuates HG-induced oxidative stress in ARPE 19 cells. (A) Cell viability was examined using a CCK-8 kit when ARPE I 9 cells were treated with different dose of DHM. (B) Cell viability was detected using a CCK-8 kit when ARPE 19 cells were stimulated with HG in the presence or absence of DHM. (C) The production of intracellular ROS was measured using DCFH-DA as a fluorescence probe. (D-F) The levels of oxidative stress-related markers including SOD, CAT and GSH were evaluated using the commercially available kits. ${ }^{* * *} \mathrm{P}<0.00$ I vs MA; ${ }^{\#} \mathrm{P}<0.05,{ }^{\#} \mathrm{P}<0.0 \mathrm{I},{ }^{\#} \mathrm{P}<0.00 \mathrm{I}$ vs $\mathrm{HG}$.

Abbreviations: DHM, Dihydromyricetin; HG, high glucose; NG, normal glucose; MA, mannitol; CCK-8, Cell Counting Kit-8; ROS, reactive oxygen species; DCFH-DA, 2', 7'-dichlorodihydrofluorescein diacetate; SOD, superoxide dismutase; CAT, catalase; GSH, glutathione. 
was employed to stimulate cells to simulate the DR model in vitro. As shown in Figure $1 \mathrm{~B}$, cell viability was notably decreased after $\mathrm{HG}$ challenge relative to the MA group and the osmotic pressure control group. By contrast, significant elevation of cell viability was observed following DHM intervention. Subsequently, the production of intracellular ROS was detected. It was observable from Figure $1 \mathrm{C}$ that $\mathrm{HG}$ stimulation triggered the marked increase of intracellular ROS compared with the MA group, while DHM dosedependently decreased it in ARPE 19 cells. Consistently, the activities of SOD, CAT antioxidases, and the content of GSH were apparently reduced after HG challenge. However, these changes in oxidative stress-related markers were dose-dependently reversed in DHM-treated group (Figure 1D-F). These results suggest that DHM attenuates $\mathrm{HG}$-induced oxidative stress in ARPE 19 cells.

\section{DHM Inhibits Apoptosis in HG-Stimulated ARPE 19 Cells}

To clarify the role of DHM in apoptosis of HG-induced ARPE 19 cells, cell apoptosis was determined using TUNEL staining. As exhibited in Figure 2A and B, HG exposure led to significant elevation in apoptotic ARPE 19 cells compared with the MA group. It was worth noting that DHM abated the impacts of HG induction on cell apoptosis in a concentrationdependent way. Additionally, results obtained from Western blot analysis presented that the expression of anti-apoptotic protein Bcl-2 was notably downregulated in the HG model group relative to the osmotic pressure control group, accompanied by upregulated expression of pro-apoptotic proteins including Bax, cleaved caspase-3 and cleaved caspase-9 (Figure 3). On the contrary, DHM pretreatment reversed the expression of above mentioned apoptosis-related proteins compared with the HG group. These observations reveal that DHM inhibits HG-induced apoptosis in ARPE 19 cells.
A
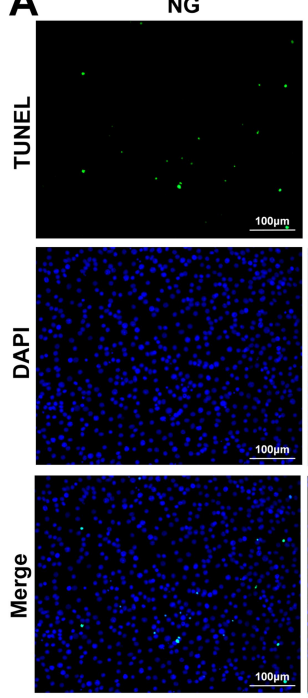

MA
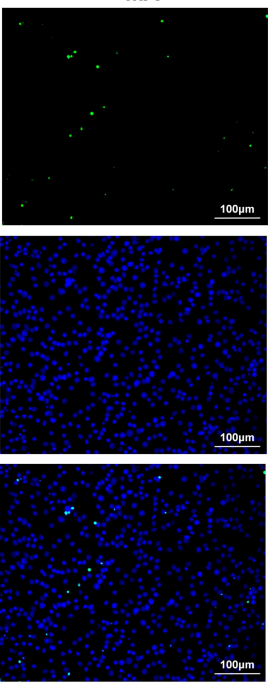

HG
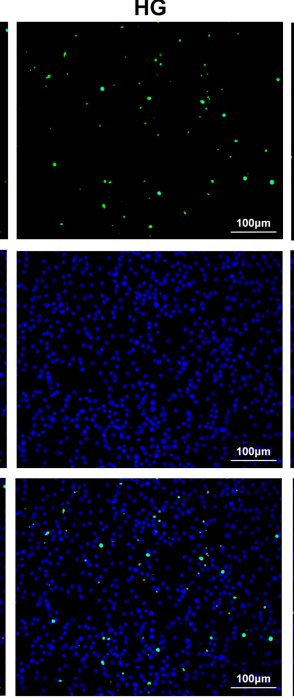

HG+30 $\mu \mathrm{mol} / \mathrm{L}$ DHM
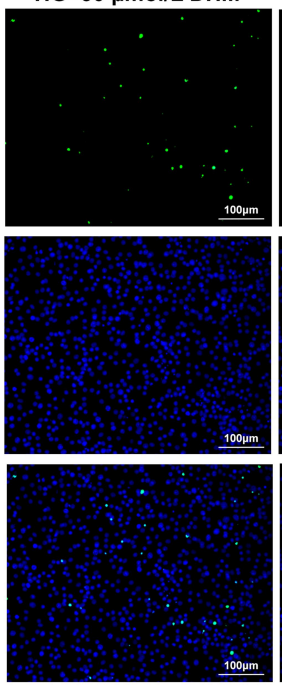

B

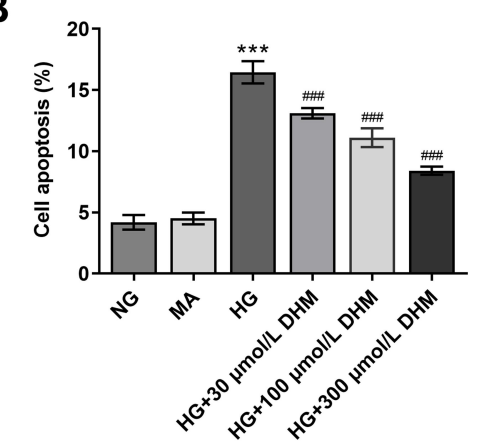

HG+100 $\mu \mathrm{mol} / \mathrm{L}$ DHM

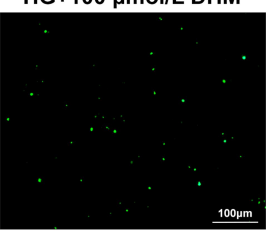

HG+300 $\mu \mathrm{mol} / \mathrm{L} \mathrm{DHM}$
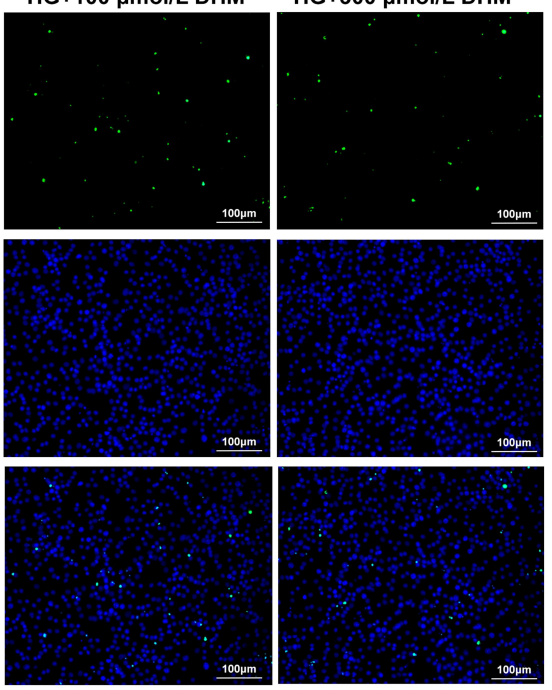

Figure 2 DHM suppresses apoptosis in HG-stimulated ARPE 19 cells. (A) Apoptosis of HG-induced ARPE 19 cells in the presence or absence of DHM was measured using

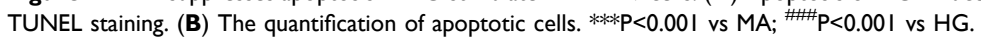

Abbreviations: DHM, Dihydromyricetin; HG, high glucose; NG, normal glucose; MA, mannitol; TUNEL, terminal-deoxynucleoitidyl transferase mediated nick end labeling (TUNEL) staining. 

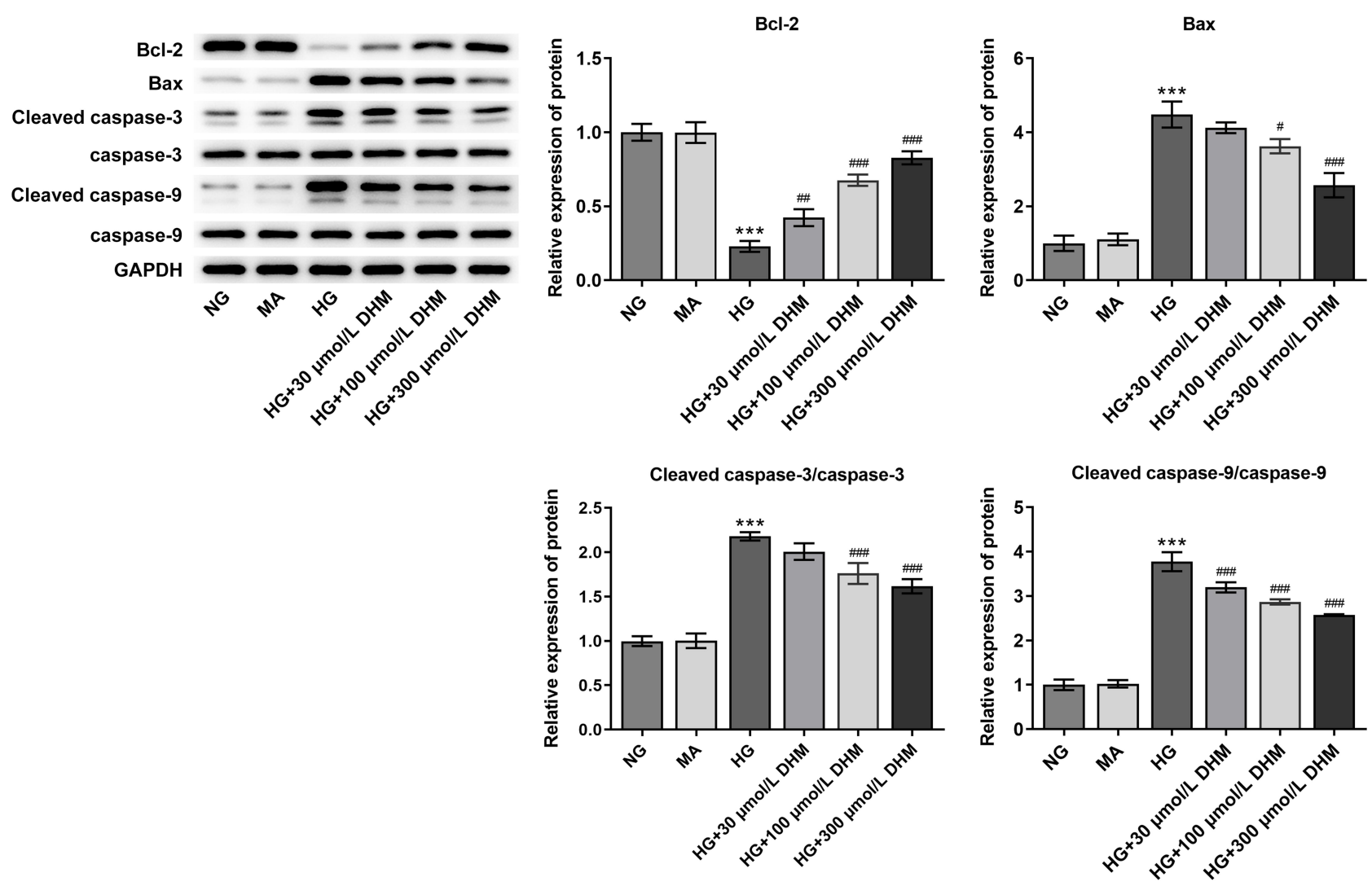

Figure 3 DHM affects the expression of apoptosis-related proteins in ARPE 19 cells exposed to HG. The expression of Bcl-2, Bax, cleaved caspase-3 and cleaved caspase-9 was evaluated using Western blot analysis. ${ }^{* * * P} \mathrm{P}<0.00$ I vs MA; ${ }^{\#} \mathrm{P}<0.05,{ }^{\#} \mathrm{P}<0.0$ I, ${ }^{\# \#} \mathrm{P}<0.00 \mathrm{I}$ vs HG.

Abbreviations: DHM, Dihydromyricetin; HG, high glucose; NG, normal glucose; MA, mannitol.

\section{MiR-34a Overexpression Restores the Inhibitory Effects of DHM on Oxidative Stress in ARPE 19 Cells Exposed to HG}

To explore the underlying regulatory mechanisms of DHM in HG-stimulated ARPE 19 cells, the expression of miR-34a was detected using RT-qPCR. Significantly enhanced miR-34a expression in ARPE 19 cells was found after HG challenge as compared to the MS group, while DHM downregulated the level of miR-34a relative to the HG group (Figure 4A). To further investigate whether DHM could work in HG-exposed ARPE 19 cells through regulation miR-34a expression, miR-34a was overexpressed by transfection with miR-34a mimic, and notably enhanced miR-34a expression was observed after transfection (Figure 4B). Afterwards, the levels of oxidative stress-associated markers were tested. As exhibited in Figure 4C-F, the elevated content of ROS and reduced levels of SOD, CAT and GSH induced by HG stimulation were remarkably restored following DHM intervention. These data provide evidence that
DHM attenuates HG-induced oxidative stress in ARPE 19 cells via inhibiting miR-34a expression.

\section{MiR-34a Overexpression Abrogates the Impact of DHM on Apoptosis in HG-Induced ARPE 19 Cells}

Subsequently, cell apoptosis was assessed after miR-34a overexpression in ARPE 19 cells exposed to HG in the presence or absence of DHM. As shown in Figure 5A and $\mathrm{B}$, there was no significant difference on cell apoptosis between DHM group and DHM plus mimic-NC group in ARPE 19 cells with HG challenge, while cell apoptosis was apparently increased after miR-34a overexpression relative to the mimic-NC group. Concurrently, overexpression of miR-34a dramatically decreased the expression level of Bcl-2 while increased those of Bax, cleaved caspase- 3 and cleaved caspase- 9 compared with the mimic control group (Figure 6). Through the above findings, we proved that DHM mitigates HG-induced apoptosis of ARPE 19 cells via inhibiting miR-34a expression. 
A

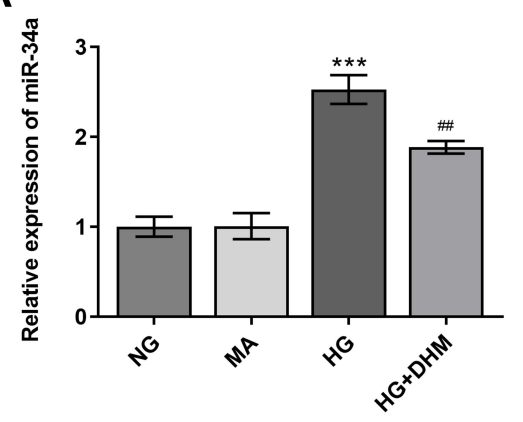

B

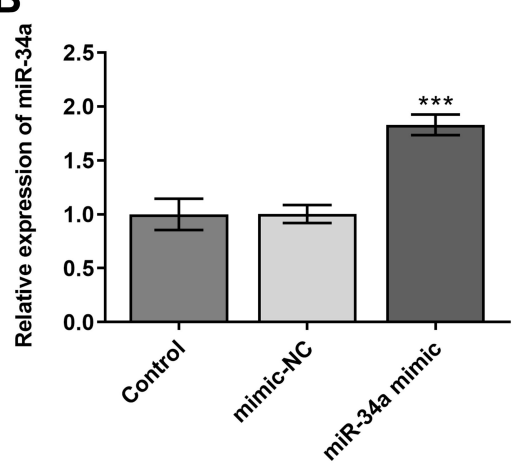

D

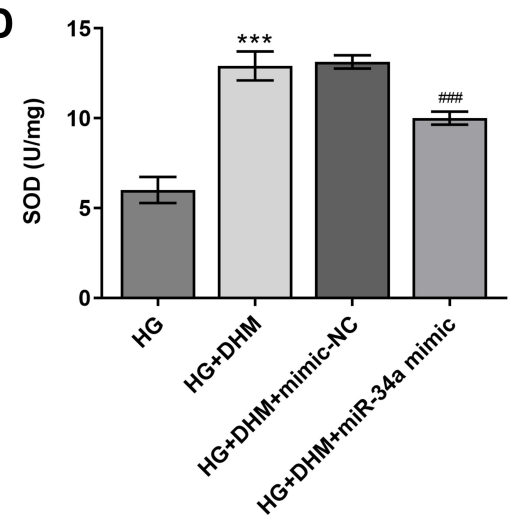

C

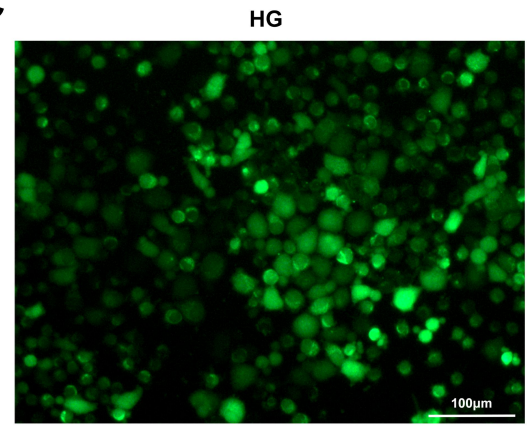

HG+DHM+mimic-NC

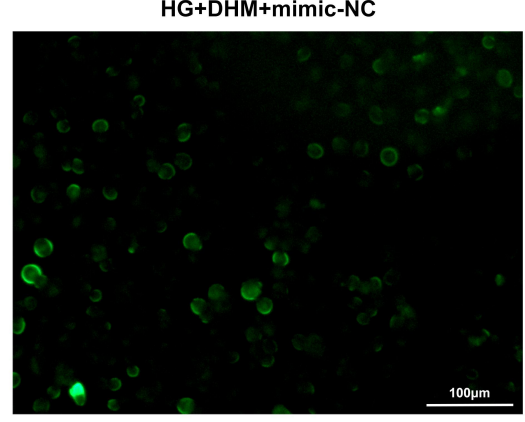

E

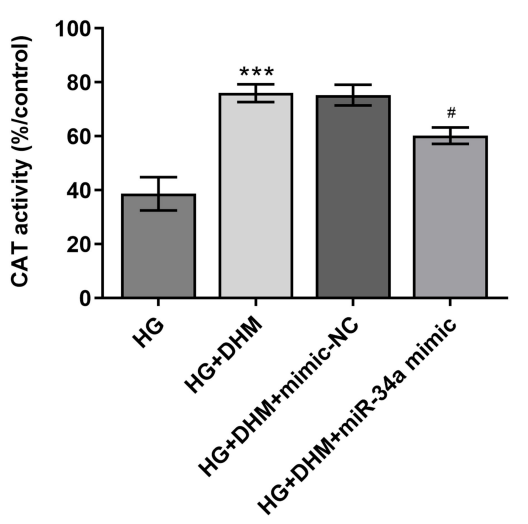

HG+DHM

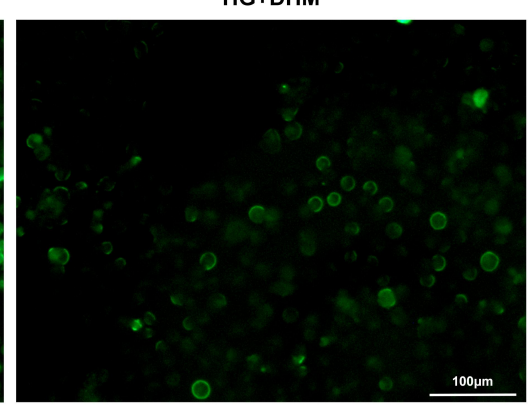

HG+DHM+miR-34a mimic
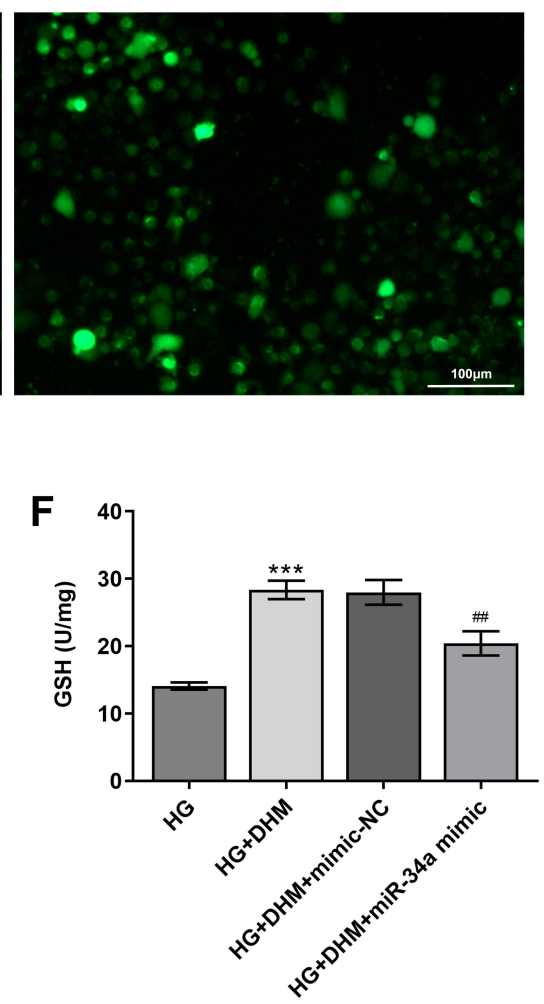

Figure 4 MiR-34a expression alleviates the inhibitory effects of DHM on oxidative stress in ARPE 19 cells exposed to HG. (A) The expression of miR-34a was determined

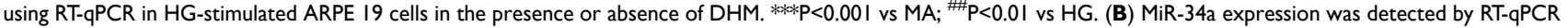
after transfection with miR-34a mimic. $* * * P<0.00 I$ vs mimic-NC. (C) Representative microscopic picture of cells emitting fluorescence of DCFH-DA. (D-F) The activities of

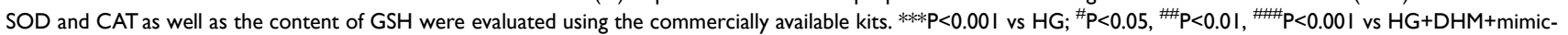
NC.

Abbreviations: DHM, Dihydromyricetin; HG, high glucose; NC, negative control; ROS, reactive oxygen species; DCFH-DA, 2', 7'-dichlorodihydrofluorescein diacetate; SOD, superoxide dismutase; CAT, catalase; GSH, glutathione.

\section{Discussion}

DR seriously endangers the health of human beings, which can lead to visual impairment and even legal blindness. ${ }^{27}$ RPE cells play particularly significant roles in the maintenance and survival of photoreceptors, and the apoptosis of the RPE related to oxidative stress. ${ }^{28} \mathrm{RPE}$ cells were widely used as in vitro models for DR research, and HGtriggered oxidative stress and apoptosis of RPE cells contributed to the occurrence and progression of DR. ${ }^{9}$ The present study demonstrated that DHM, a natural flavanol compound isolated from Ampelopsis grossedentata, could protect ARPE 19 cells from oxidative stress and apoptosis by downregulating miR-34a expression in high-glucose environments.

Mounting evidence has supported that diabetesassociated hyperglycaemia can result in a rise in ROS production within the retina, which is considered as a major causative factor for DR development. ${ }^{29,30}$ ROS increases the chronological age of cells and reduces the mitochondrial function in RPE cells, causing cell damage. 


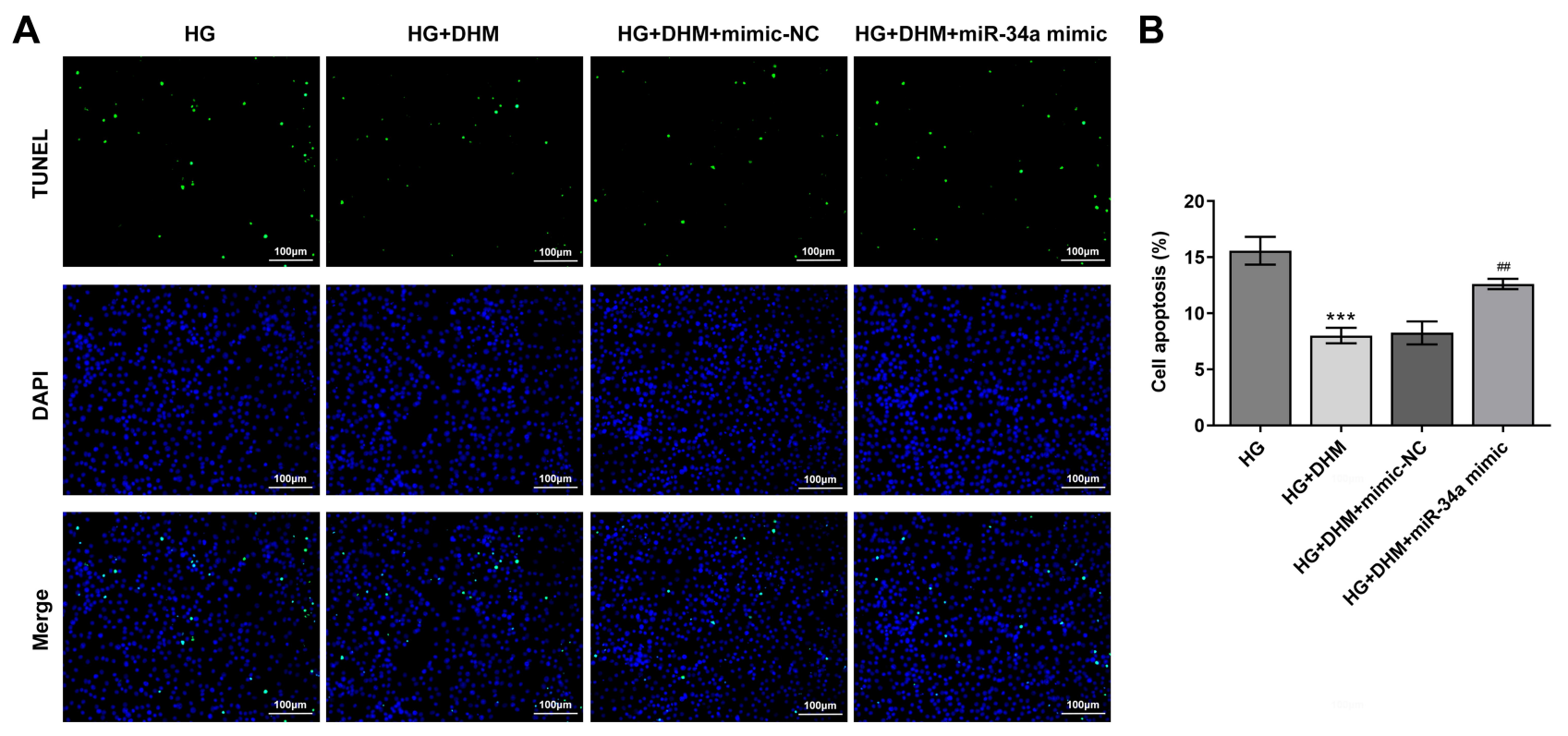

Figure 5 MiR-34a overexpression blocks the impact of DHM on apoptosis in HG-induced ARPE 19 cells. (A) Representative images of cell apoptosis. (B) The quantification of apoptotic cells. ${ }^{* * *} \mathrm{P}<0.00 \mathrm{I}$ vs $\mathrm{HG}$; ${ }^{\#} \mathrm{P}<0.0 \mathrm{I}$ vs $\mathrm{HG}+\mathrm{DHM}+$ mimic-NC.

Abbreviations: DHM, Dihydromyricetin; HG, high glucose; NG, normal glucose; NC, negative control.

Repeated cell exposure to oxidative stress leads to the insufficient activity of cellular antioxidant enzymes responding to ROS, contributing to the inability of RPE cells to prevent the consequent damages. ${ }^{31}$ Excess intracellular ROS brings about the damage of macromolecules and acts as cellular messengers to activate a cascade of biochemical and molecular events and disrupt the normal mechanisms of cellular signaling, which leads to the disruption of normal retinal cellular physiology and apoptosis. ${ }^{12,13}$ Therefore, anti-oxidation and anti-apoptosis may represent a promising therapy for the treatment of DR. DHM, also known as ampelopsin, is a dihydroflavonol flavonoid that is found in ampelopsis. A previous study has proven that DHM prevents fatty liver via inhibiting oxidative stress and apoptosis in a fatty liver rat model..$^{32}$ DHM alleviates oleic acid-induced lipid accumulation in LO2 and HepG2 cells by suppressing lipogenesis and oxidative stress. ${ }^{33}$ DHM ameliorates oxidative stress and apoptotic damage of human umbilical vein endothelial cells induced by sodium nitroprusside. ${ }^{18}$ It is noteworthy that DHM protects against diabetic cardiomyopathy and diabetic nephropathy. ${ }^{21,22}$ In this study, the significant inhibitory effects of DHM on HG-induced oxidative stress and apoptosis in ARPE 19 cells was observed, suggesting the protective effects of DHM on DR.

Aberrant expressions of multiple miRNAs are closely related to oxidative stress and DR development. ${ }^{34,35}$ It is worthy of note that miR-34a was over-expressed in Type 2 diabetes mellitus and diabetes mellitus patients, and sequence variation in miR-34a is associated with diabetes mellitus susceptibility in a southwest Chinese Han population. ${ }^{36}$ A growing body of literature has shown that miR-34a is involved in the occurrence and progression of diabetes complications, such as diabetic cardiomyopathy, diabetes mellitus-induced vascular endothelial dysfunction and diabetic nephropathy. ${ }^{37-39}$ Emerging evidence supports that miR-34a was high expression in HG-induced retina epithelial cells, and miR-34a knockdown could suppress HG-induced apoptosis and secretion of inflammation cytokines. ${ }^{40}$ Of note, DHM was reported to prevent diabetic cardiomyopathy via miR-34a suppression by activating autophagy. ${ }^{21}$ Therefore, whether the functions of DHM works in HG-exposed ARPE 19 cells through regulation of miR-34a expression was investigated in this study, and the findings demonstrated that miR-34a blocks the inhibitory effects of DHM on oxidative stress and apoptosis in ARPE 19 cells after HG exposure.

\section{Conclusion}

Taken together, these investigations for the first time delineated that DHM protects RPE cells from HG-induced oxidative stress and apoptosis. The protective effects are mediated by the downregulation of miR-34a expression. These findings corroborated that DHM could possess 

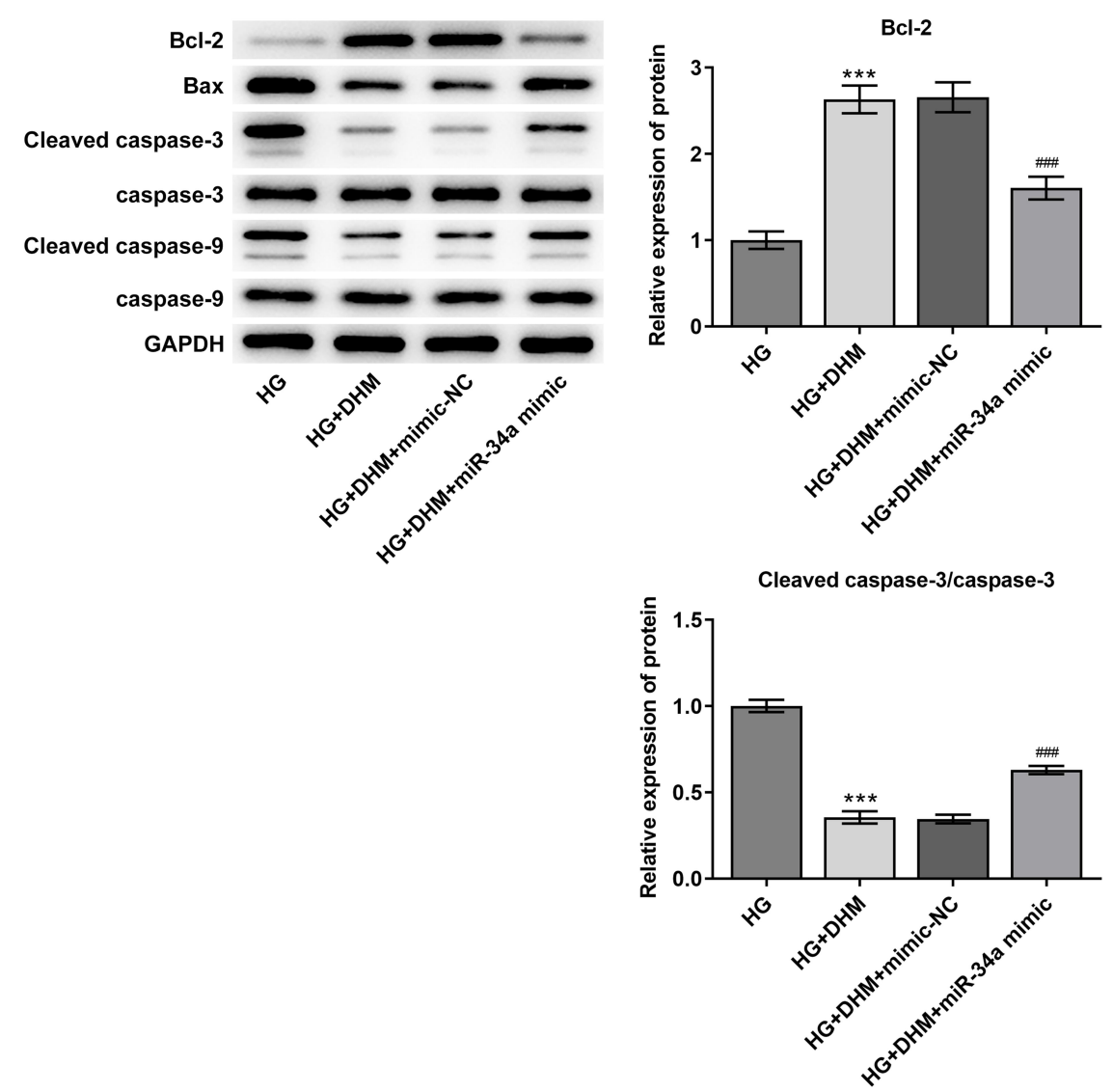

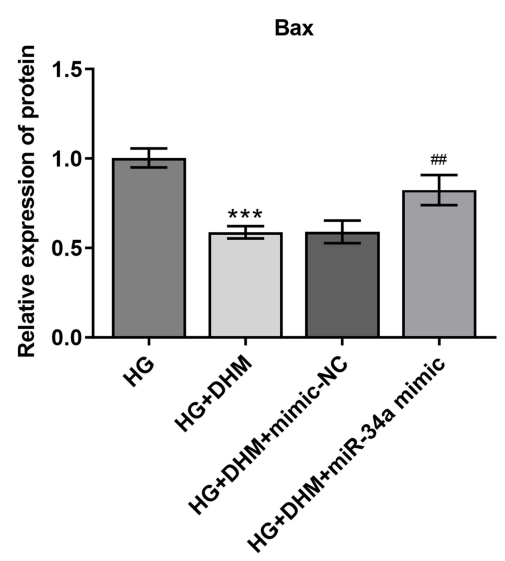

Cleaved caspase-9/caspase-9

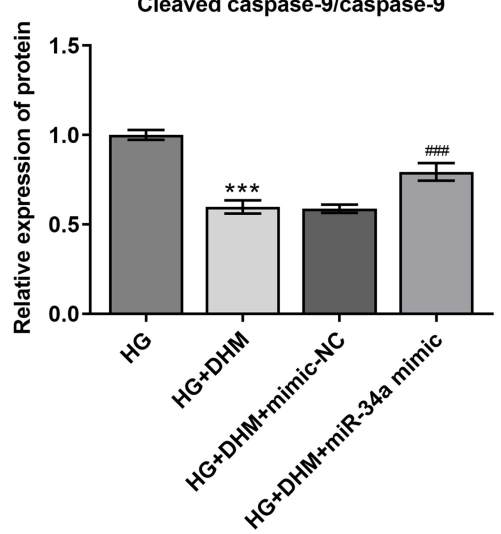

Figure 6 MiR-34a-upregulation recovers the effects of DHM on the expression of apoptosis-related proteins in ARPE 19 cells exposed to HG. The expression of Bcl-2, Bax, cleaved caspase- 3 and cleaved caspase- 9 was evaluated using Western blot analysis. ${ }^{* * * P}<0.00$ I vs HG; ${ }^{\# \#} \mathrm{P}<0.01,{ }^{\# \#} \mathrm{P}<0.00 \mathrm{I}$ vs $\mathrm{HG}+\mathrm{DHM}+\mathrm{mimic}-\mathrm{NC}$.

Abbreviations: DHM, Dihydromyricetin; HG, high glucose; NG, normal glucose; NC, negative control.

a therapeutic significance in the treatment and prevention of DR, offering experimental and scientific evidence for the potential of using DHM as a therapeutic agent in clinical DR patients. However, the lack of study in vivo is a limitation of the present research. Therefore, further experiments will be performed in the future investigations.

\section{Funding}

This study was supported by Jingmen Science and Technology Research and Development Project (2019YFYB018).

\section{Disclosure}

The authors declare no conflict of interest for this work.

\section{References}

1. Murchison AP, Hark L, Pizzi LT, et al. Non-adherence to eye care in people with diabetes. BMJ Open Diabetes Res Care. 2017;5(1): e000333. doi:10.1136/bmjdrc-2016-000333

2. Huang Y, Qian C, Zhou J, Xue J. Investigation of expression and influence of CTGF and HO-1 in rats with diabetic retinopathy. Exp Ther Med. 2020;19(3):2291-2295. doi:10.3892/etm.2019.8395
3. Pedersen BK. Anti-inflammatory effects of exercise: role in diabetes and cardiovascular disease. Eur J Clin Invest. 2017;47(8):600-611. doi:10.1111/eci.12781

4. Abu El-Asrar AM, Midena E, Al-Shabrawey M, Mohammad G. New developments in the pathophysiology and management of diabetic retinopathy. J Diabetes Res. 2013;2013:424258. doi:10.1155/2013/ 424258

5. Gong X, Draper CS, Allison GS, Marisiddaiah R, Rubin LP. Effects of the macular carotenoid lutein in human retinal pigment epithelial cells. Antioxidants (Basel). 2017;6(4). doi:10.3390/antiox6040100

6. Chang YC, Lin CW, Hsieh MC, et al. High mobility group B1 up-regulates angiogenic and fibrogenic factors in human retinal pigment epithelial ARPE-19 cells. Cell Signal. 2017;40:248-257. doi:10.1016/j.cellsig.2017.09.019

7. Ponnalagu M, Subramani M, Jayadev C, Shetty R, Das D. Retinal pigment epithelium-secretome: a diabetic retinopathy perspective. Cytokine. 2017;95:126-135. doi:10.1016/j.cyto.2017.02.013

8. Bahrami B, Shen W, Zhu L, Zhang T, Chang A, Gillies MC. Effects of VEGF inhibitors on human retinal pigment epithelium under high glucose and hypoxia. Clin Exp Ophthalmol. 2019;47(8):1074-1081. doi:10.1111/ceo.13579

9. Tenconi PE, Bermudez V, Oresti GM, Giusto NM, Salvador GA, Mateos MV. High glucose-induced phospholipase D activity in retinal pigment epithelium cells: new insights into the molecular mechanisms of diabetic retinopathy. Exp Eye Res. 2019;184:243-257. doi:10.1016/j.exer.2019.04.028

10. Behl T, Kaur I, Kotwani A. Implication of oxidative stress in progression of diabetic retinopathy. Surv Ophthalmol. 2016;61 (2):187-196. doi:10.1016/j.survophthal.2015.06.001 
11. Kowluru RA, Mishra M. Oxidative stress, mitochondrial damage and diabetic retinopathy. Biochim Biophys Acta. 2015;1852 (11):2474-2483. doi:10.1016/j.bbadis.2015.08.001

12. Cunha-Vaz J. Mechanisms of retinal fluid accumulation and blood-retinal barrier breakdown. Dev Ophthalmol. 2017;58:11-20. doi: $10.1159 / 000455265$

13. Xiao H, Liu Z. Effects of microRNA217 on high glucose induced inflammation and apoptosis of human retinal pigment epithelial cells (ARPE19) and its underlying mechanism. Mol Med Rep. 2019;20 (6):5125-5133. doi:10.3892/mmr.2019.10778

14. Wang Y, Zhou L, Li R, Wang Y. [Determination of ampelopsin in the different parts of Ampelopsis grossedentata in different seasons by RP-HPLC]. Zhong Yao Cai. 2002;25(1):23-24. Chinese.

15. Hou X, Tong Q, Wang W, Xiong W, Shi C, Fang J. Dihydromyricetin protects endothelial cells from hydrogen peroxide-induced oxidative stress damage by regulating mitochondrial pathways. Life Sci. 2015;130:38-46. doi:10.1016/j.1fs.2015.03.007

16. Liu CM, Yang W, Ma JQ, et al. Dihydromyricetin inhibits leadinduced cognitive impairments and inflammation by the adenosine 5 '-monophosphate-activated protein kinase pathway in mice. J Agric Food Chem. 2018;66(30):7975-7982. doi:10.1021/acs. jafc. $8 \mathrm{~b} 02433$

17. Zhang Q, Liu J, Liu B, et al. Dihydromyricetin promotes hepatocellular carcinoma regression via a p53 activation-dependent mechanism. Sci Rep. 2015;4(1):4628. doi:10.1038/srep04628

18. Zhang XY, Wang LF, Peng LZ, et al. Dihydromyricetin protects HUVECs of oxidative damage induced by sodium nitroprusside through activating PI3K/Akt/FoxO3a signalling pathway. $J$ Cell Mol Med. 2019;23(7):4829-4838. doi:10.1111/jcmm.14406

19. Jiang BP, Le L, Pan HM, Hu KP, Xu LJ, Xiao PG. Dihydromyricetin ameliorates the oxidative stress response induced by methylglyoxal via the AMPK/GLUT4 signaling pathway in PC12 cells. Brain Res Bull. 2014;109:117-126. doi:10.1016/j.brainresbull.2014.10.010

20. Liu L, Zhou M, Lang HD, Zhou Y, Mi MT. Dihydromyricetin enhances glucose uptake by inhibition of MEK/ERK pathway and consequent down-regulation of phosphorylation of PPAR in 3T3-L1 cells. J Cell Mol Med. 2018;22(2):1247-1256. doi:10.1111/ jcmm.13403

21. Ni TJ, Lin N, Lu WQ, et al. Dihydromyricetin prevents diabetic cardiomyopathy via mir-34a suppression by activating autophagy. Cardiovasc Drugs Ther. 2020;34(3):291-301. doi:10.1007/s10557020-06968-0

22. Guo LM, Tan KB, Luo Q, Bai X. Dihydromyricetin promotes autophagy and attenuates renal interstitial fibrosis by regulating miR-1555p/PTEN signaling in diabetic nephropathy. Bosnian J Basic Med Sci. 2020;20(3):372-380.

23. Luo R, Jin H, Li L, Hu YX, Xiao F. Long noncoding RNA MEG3 inhibits apoptosis of retinal pigment epithelium cells induced by high glucose via the miR-93/Nrf2 axis. Am J Pathol. 2020;190 (9):1813-1822. doi:10.1016/j.ajpath.2020.05.008

24. Martinez B, Peplow PV. MicroRNAs as biomarkers of diabetic retinopathy and disease progression. Neural Regen Res. 2019;14 (11):1858-1869. doi:10.4103/1673-5374.259602

25. Wu JD, Liang WZ, Tian YL, et al. Inhibition of P53/miR-34a improves diabetic endothelial dysfunction via activation of SIRT1. J Cell Mol Med. 2019;23(5):3538-3548. doi:10.1111/jcmm.14253
26. Thounaojam MC, Jadeja RN, Warren M, et al. MicroRNA-34a (miR-34a) mediates retinal endothelial cell premature senescence through mitochondrial dysfunction and loss of antioxidant activities. Antioxidants (Basel). 2019;8(9). doi:10.3390/antiox8090328.

27. Cheung N, Mitchell P, Wong TY. Diabetic retinopathy. Lancet. 2010;376(9735):124-136. doi:10.1016/S0140-6736(09)62124-3

28. Yang QS, Li SZ, Zhou ZX, et al. HDAC6 inhibitor Cay10603 inhibits high glucose-induced oxidative stress, inflammation and apoptosis in retinal pigment epithelial cells via regulating NF-kappa B and NLRP3 inflammasome pathway. Gen Physiol Biophys. 2020;39 (2):169-177. doi:10.4149/gpb_2019058

29. Calderon GD, Juarez OH, Hernandez GE, Punzo SM, De la Cruz ZD. Oxidative stress and diabetic retinopathy: development and treatment. Eye (Lond). 2017;31(8):1122-1130. doi:10.1038/eye.2017.64

30. Li C, Miao X, Li F, et al. Oxidative stress-related mechanisms and antioxidant therapy in diabetic retinopathy. Oxid Med Cell Longev. 2017;2017:9702820. doi:10.1155/2017/9702820

31. Lai TT, Yang CM, Yang CH. Astaxanthin protects retinal photoreceptor cells against high glucose-induced oxidative stress by induction of antioxidant enzymes via the PI3K/Akt/Nrf2 pathway. Antioxidants (Basel). 2020;9(8). doi:10.3390/antiox9080729

32. Guo L, Zhang HF, Yan XP. Protective effect of dihydromyricetin prevents fatty liver through nuclear factor-B/p53/B-cell lymphoma 2 -associated X protein signaling pathways in a rat model. Mol Med Rep. 2019;19(3):1638-1644. doi:10.3892/mmr.2018.9783

33. Xie CF, Chen Z, Zhang CF, et al. Dihydromyricetin ameliorates oleic acid-induced lipid accumulation in L02 and HepG2 cells by inhibiting lipogenesis and oxidative stress. Life Sci. 2016;157:131-139. doi:10.1016/j.lfs.2016.06.001

34. Chen P, Miao Y, Yan P, Wang XJ, Jiang C, Lei Y. MiR-455-5p ameliorates HG-induced apoptosis, oxidative stress and inflammatory via targeting SOCS3 in retinal pigment epithelial cells. $J$ Cell Physiol. 2019;234(12):21915-21924. doi:10.1002/jcp.28755

35. Xi XT, Yang YN, Ma J, et al. MiR-130a alleviated high-glucose induced retinal pigment epithelium (RPE) death by modulating TNF-alpha/SOD1/ROS cascade mediated pyroptosis. Biomed Pharmacother. 2020;125:9. doi:10.1016/j.biopha.2020.109924

36. Sun Y, Peng R, Li AL, et al. Sequence variation in microRNA-34a is associated with diabetes mellitus susceptibility in a southwest Chinese Han population. Int J Clin Exp Pathol. 2018;11(3):1637.

37. Gao L, Wang XF, Guo S, et al. LncRNA HOTAIR functions as a competing endogenous RNA to upregulate SIRT1 by sponging miR-34a in diabetic cardiomyopathy. J Cell Physiol. 2019;234 (4):4944-4958. doi:10.1002/jcp.27296

38. Zhao D, Wang NS, Chen F, Li ZB, Li XT, Zhu XX. Intravenous injection of miR-34a inhibitor alleviates diabetes mellitus-induced vascular endothelial dysfunction by targeting NOTCH1. Exp Clin Endocrinol Diabetes. 2019;127(4):255-262. doi:10.1055/s-0043125324

39. Zhang XY, Song SP, Luo HX. Regulation of podocyte lesions in diabetic nephropathy via miR-34a in the notch signaling pathway. Medicine. 2016;95(44):6.

40. Tong P, Peng QH, Gu LM, Xie WW, Li WJ. LncRNA-MEG3 alleviates high glucose induced inflammation and apoptosis of retina epithelial cells via regulating miR-34a/SIRT1 axis. Exp Mol Pathol. 2019;107:102-109. doi:10.1016/j.yexmp.2018.12.003 


\section{Publish your work in this journal}

Diabetes, Metabolic Syndrome and Obesity: Targets and Therapy is an international, peer-reviewed open-access journal committed to the rapid publication of the latest laboratory and clinical findings in the fields of diabetes, metabolic syndrome and obesity research. Original research, review, case reports, hypothesis formation, expert opinion and commentaries are all considered for publication. The manuscript management system is completely online and includes a very quick and fair peer-review system, which is all easy to use. Visit http://www.dovepress.com/testimonials.php to read real quotes from published authors.

Submit your manuscript here: https://www.dovepress.com/diabetes-metabolic-syndrome-and-obesity-targets-and-therapy-journal 